\title{
CATASTRO DE VERTEBRADOS TERRESTRES Y ANALISIS EN SEIS HABITATS PRESENTES EN LA RESERVA NACIONAL FUTALEUFU, PROVINCIA DE PALENA, X REGION, CHILE
}

\section{TERRESTRIAL VERTEBRATE BASELINE AND ANALYSIS IN SIX HABITATS PRESENT IN THE NATIONAL RESERVE FUTALEUFU, PALENA PROVINCE, X REGION, CHILE}

\author{
Emma Elgueta ${ }^{1}$, Sharon Reid $^{1,2}$, Patricio Pliscoff ${ }^{1}$, Marco A. Méndez $^{2,3}$, \\ José Núnez ${ }^{4} \&$ Cecilia Smith-Ramírez ${ }^{1,2}$ \\ 'Fundación Senda Darwin. Casilla 114-D, CP 6513677, Santiago. Email: info@ sendadarwin.cl, \\ sendadarwin@bio.puc.cl. \\ ${ }^{2}$ Centro de Estudios Avanzados en Ecología y Biodiversidad, Pontificia Universidad Católica de Chile, \\ Casilla 114-D, Santiago. \\ ${ }^{3}$ Laboratorio de Bioinformática y Expresión Génica (INTA). Universidad de Chile. Macul 5540, Santiago. \\ ${ }^{4}$ Instituto de Zoología, Facultad de Ciencias. Universidad Austral de Chile. Casilla 567 Valdivia.
}

\begin{abstract}
RESUMEN
Se realizó un catastro de los mamíferos, aves, reptiles y anfibios presentes en seis hábitats de la Reserva Nacional Futaleufú, provincia de Palena, Chile. Estos hábitats se encuentran desde la cota 700 hasta los 2000 msnm, ellos son: cipresal, matorral mixto, bosque de lenga y coigüe, renoval de Nothofagus, mallín y bosque achaparrado de ñirres en sectores altos de roqueríos con nieves estacionales. Se registraron un total de 60 especies de vertebrados en la Reserva: 17 especies de mamíferos, 38 especies de aves, cuatro especies de anfibios y una especie de reptil. Se destaca la presencia de huemules en casi todos los hábitats. El análisis por hábitat muestra una mayor riqueza de especies en los bosques de lenga-coigüe, los cuales presentan además una mayor heterogeneidad estructural. El matorral mixto, que limita con praderas y campos agrícolas, y los renovales de Nothofagus representan el segundo y tercer hábitat con mayor riqueza. Se advierte la fragilidad del sistema para mantener poblaciones de vertebrados, pues hacia el borde de la Reserva se produce una interfase entre el uso por animales domésticos (perros, ganado y caballos) y la fauna silvestre endémica o con problemas de conservación.
\end{abstract}

Palabras ClaVEs: Biodiversidad, áreas silvestres protegidas, fauna patagónica.

\section{ABSTRACT}

We surveyed the mammals, birds, reptiles and amphibians in six distinct habitat types in the Futaleufú National Reserve, in the province of Palena, Chile. These habitats occur from 700 masl up to 2.000 masl: cypress forest, mixed shrubland, lenga and coigüe forest, early successional Nothofagus tree areas, scrubland and ñirre trees in rocky areas with seasonal snow. A total of 60 species were recorded in the Reserve: 17 terrestrial mammals, 38 bird species, four amphibians and one reptile species. We highlight the presence of huemules in almost all the habitats. Habitat type analysis showed that lenga-coigüe forests have the highest species richness for all taxa, besides having also a higher structural heterogeneity. The mixed shrubland, which limits with pastures and agriculture fields, together with the early successional Nothofagus tree areas represent the second and third habitats with highest species richness. We warn of the fragility of the system for the maintenance of vertebrate populations, because towards the edge of the Reserve, an interface results between the use by domestic animals (dogs, livestock and horses) and the wild endemic fauna or with conservation problems.

KEYwords: Biodiversity, wildlife reserve areas, Patagonic fauna. 


\section{INTRODUCCION}

La identificación de áreas geográficas adecuadas para la conservación de la fauna debiera basarse en la caracterización local de la riqueza, endemismo y/o grado de amenaza de las especies que la habitan. Esto ha sido aplicado mundialmente en la elección de áreas protegidas para escoger los llamados "hotspot", o áreas de interés para la conservación mundial (Prendergast 1993, Simonetti 1999, Myers et al. 2000). Sin embargo estas aproximaciones muestran dificultades para ser aplicadas en América latina, incluyendo Chile, dado que la base de datos sobre la distribución geográfica y abundancia de los taxa es bastante incompleta (Cofré \& Marquet 1999; Primack et al. 2001). Por esta razón, estudios locales de biodiversidad en áreas de pequeño y mediano tamaño permiten evaluar la efectividad y factibilidad del trabajo de conservación en estas áreas. En especial si consideramos como área de interés las reservas ya establecidas no siempre con un criterio de mayor riqueza, endemismo y presencia de especies amenazadas, en que además ya existe un historial de uso e interacción entre gente local y flora/fauna nativa. Por otro lado, las características particulares de cada área a proteger, como la heterogeneidad de hábitats (Freemark \& Merriam 1986, Kerr \& Paker 1997) entregan un elemento más a considerar en el diseño de áreas para la conservación de la biodiversidad.

La Reserva Nacional Futaleufú se encuentra en la Cordillera de los Andes de la X Región y va desde la cota de los $700 \mathrm{msnm}$ hasta los $2.000 \mathrm{msnm}$, generando una fuerte gradiente altitudinal con distintas franjas de formaciones vegetacionales: cipresales; bosques de lenga y coigüe en distintos grados de regeneración; matorral siempreverde y bosques achaparrados de ñirres. Formaciones vegetales que sumadas a las variaciones geomorfológicas del lugar, como acantilados y altas cumbres con nieve gran parte del año, brindan hábitats de distintivas características estructurales en un área relativamente pequeña. La creación de esta área silvestre protegida, el año 1998, tuvo por objetivo principal la protección de una especie, el huemul (Hippocamelus bisulcus Molina). Posterior a la creación de la Reserva existió la inquietud por la Corporación Nacional Forestal de conocer el potencial de conservación de vertebrados más allá de la especie focal que llevó a su creación. Es por esto que los objetivos de este trabajo son entregar un catastro de los vertebrados terrestres (mamíferos, aves, reptiles y anfibios) presentes en la Reserva y describir la distribución de la riqueza faunística por hábitats. Además, se identifican los sectores que albergan las especies con problemas de conservación y endémicas a los bosques templados sudamericanos.

\section{MATERIALES Y METODOS}

Area De estudio

La Reserva Nacional Futaleufú (12.065 hectáreas), se encuentra en la comuna de Futaleufú, provincia de Palena, X Región de Chile ( $43^{\circ} 17^{\prime}$ - 43 $37^{\circ}$ 'S y $71^{\circ} 73^{\prime}-71^{\circ} 91^{\prime} \mathrm{W}$, Figura 1). Posee un clima lluvioso templado húmedo durante todo el año $(\mathrm{Cfb})$, con temperatura promedio de $10,1^{\circ} \mathrm{C}$ y precipitaciones superiores a los $2.000 \mathrm{~mm}$ (Di Castri \& Hayek 1976). Geográficamente se encuentra inserta en los macizos cordilleranos andinos en la frontera con Argentina, presentando un abrupto relieve que va desde las zonas bajas de los valles formados por tributarios del río Futaleufú, hasta la presencia de cumbres que superan los 2.000 metros de altitud

Con el objetivo de caracterizar a nivel local la diversidad de vertebrados de la Reserva, se consideró necesario subdividir la Reserva dentro de hábitats o biotopos, de tal forma de poder realizar un análisis lo más detallado posible y comparable a futuro con otras áreas donde pudieran existir estos hábitats.

Se definieron seis tipos de hábitats dentro de la Reserva, de acuerdo a la dominancia arbórea de las principales formaciones vegetacionales, su estado de sucesión y las formaciones geológicas presentes. Estos hábitats se encuentran representados en los polígonos de la cartografía diseñada para este estudio (Figura 1): Estos hábitats son: (1) bosquetes de ciprés de la cordillera (Pilgerodendrum uviferum (D. Don) Florin) en la cota inferior; (2) matorral mixto de maqui (Aristotelia chilensis Mol. Stuntz), canelo (Drimys winteri J.R. Forst et G. Forst.) y mirtáceas creciendo en asociación con especies introducidas desde las praderas; (3) bosques de lenga (Nothofagus pumilio (Poepp. et Endl.) Krasser) y coigüe de Magallanes (Nothofagus betuloides (Mirb.) Oerst.) en distintos grados de 
intervención; (4) Renoval de Nothofagus derivados de incendios anteriores; (5) pajonal o mallín en el origen del río Correntoso cubierto por especies de Scirpus sp. y plantas en cojín; y (6) terrenos rocosos de las partes altas de la Reserva (sobre $1.600 \mathrm{msnm}$ ) que son cubiertos por nieve estacionalmente y donde esporádicamente crecen bosquetes de ñirre (Nothofagus antarctica (G.Forst.) Oerst). Todos los hábitats conforman extensiones sobre las 1.000 hectáreas, con un máximo de 7.000 ha (aprox.) para el hábitat de bosques de lenga-coigüe y un mínimo de 1.000 ha (aprox.) para el mallín.

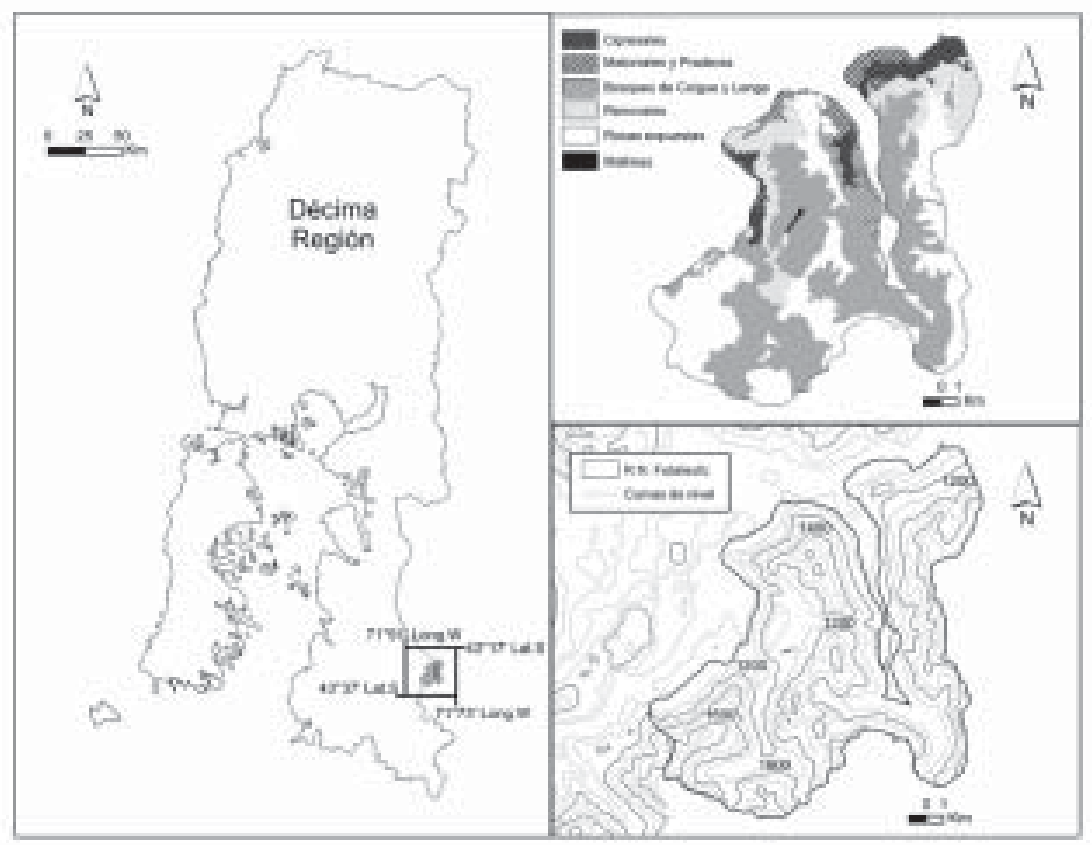

FIgURA 1. Ubicación geográfica y topografía de la Reserva Nacional Futaleufú en la X Región. La cartografía dentro de la Reserva marca los hábitats de mayor extensión en los cuales se realizaron los muestreos.

FIGURE 1. Geographic location and topography of the Futaleufú National Reserve in the 10th Region. The cartography inside the Reserve marks the habitats with the largest area in which surveys were conducted.

\section{MÉTOdo DE MUESTREO}

Durante la segunda semana de enero del 2003 se determinó la composición faunística de mamíferos, aves, reptiles y anfibios, basados en evidencias directas e indirectas dentro de los seis hábitats definidos para la Reserva.

MAMífERos: En cada sector visitado (excepto sobre mallines y acantilados) se estableció un transecto con 10 estaciones olfativas ubicadas cada 100 metros entre sí. Las estaciones olfativas se establecieron con el atrayente comercial para carnívoros "bobcat urine” y para los cérvidos se usó el atrayente comercial "whitetail deer". Se recolectaron y analizaron fecas de carnívoros y egagrópilas de aves rapaces para determinar la presencia de micromamíferos mediante la comparación de los molares encontrados con la clave de Pearson (1995). Se determinó la presencia de Quirópteros mediante la observación a ojo desnudo y por binoculares a las horas crepusculares. Adicionalmente se determinó de manera indirecta la presencia de mamíferos en base a recolecta de fecas y discriminación de huellas frescas, galerías y dormideros en uso (Iriarte et al. 1989; Martínez et al. 1993) y el registro sistemático de los guardaparques. 
Aves: La estimación de la riqueza y abundancia de la avifauna se realizó mediante estaciones de escucha cada mañana entre las 07:30 y 09:00 hrs. (Ralph et al. 1993; Willson et al. 1994), en los seis tipos de hábitat. En cada estación, se registraron todas las aves (contacto visual o auditivo) dentro de un área de radio $25 \mathrm{~m}$., durante un período de 5 minutos por estación (Willson et al. 1994). Las estaciones de muestreo estaban a más de $150 \mathrm{~m}$ de distancia entre sí para minimizar el riesgo de contar un mismo individuo dos veces (Hutto et al. 1986). Para completar la lista de especies se registraron durante todo el día todas las especies no detectadas en los censos por cada hábitat. Como evidencia indirecta se buscaron fecas, egagrópilas, plumas, nidos, restos de esqueletos y huevos.

ANFibIos y RePtiLes: Para los anfibios y reptiles se realizó una búsqueda exhaustiva de las especies presentes a lo largo de un transecto latitudinal y altitudinal en los mismos hábitats muestreados para los otros taxa. Se buscaron anfibios y reptiles entre y bajo la hojarasca, a orillas de ríos, bajo piedras y troncos, así como también se realizaron escuchas especialmente a la puesta del sol. En el caso de los reptiles se buscó especialmente en los lugares expuestos al sol.

Las categorías de conservación para las especies encontradas se determinaron de acuerdo a la lista oficial emitida por el SAG, en la edición, 2001 de la Cartilla de Caza de acuerdo al DS del Reglamento de la Ley de Caza publicado en el Diario Oficial el 7 de diciembre de 1998.

\section{RESULTADOS}

Considerados los registros directos e indirectos, la Reserva Nacional Futaleufú contaría al menos con 60 especies de vertebrados: 17 especies de mamíferos silvestres, 38 especies de aves, cuatro especies de anfibios y una especie de reptil (Tabla I).

Mamíreros: Se identificaron al menos 17 especies de mamíferos nativos por evidencia directa e indirecta (Tabla I) pertenecientes a cinco órdenes: Quiroptera (2), Rodentia ( $\left.7^{1}\right)$, Carnivora (5), Edentata (1) y Artiodactyla (2). Los mamíferos terrestres que aparecen utilizando casi todos los hábitats de la Reserva son el huemul y el zorro culpeo, incluso en terrenos muy cercanos a los asentamientos humanos donde se acercan en busca de agua. De los análisis de los molares encontrados en fecas y egagrópilas al menos seis especies de micromamíferos se describen para toda la Reserva (Tabla I). En las zonas bajas los registros de molares y huesos en fecas de zorros correspondieron en su mayoría a restos de liebres. Respecto a evidencias indirectas, en el hábitat de rocas expuestas, con presencia de bosque achaparrado de ñirre, se encontraron huellas de un felino que hemos considerado como Oncifelis sp.

Respecto a las categorías de conservación de las especies registradas cuatro son catalogadas como en Peligro de extinción (SAG 2001): el huemul (Hippocamelus bisulcus Molina), la huiña (Oncifelis guigna Molina), el zorro culpeo (Pseudalopex culpaeus Molina) (categoría específica para la zona) y la vizcacha (Lagidium viscacia Molina); y tres especies están en la categoría de Vulnerable a la extinción: el puma (Puma concolor Linnaeus), el pudú (Pudu puda Molina) y el murciélago oreja de ratón (Myotis chiloensis Waterhouse), estos dos últimos son además endémicos al bosque templado. El quirquincho de la Patagonia o piche (Zaedyus pichiy Desmarest) es considerado una especie Rara por lo que poblaciones de baja densidad son igualmente vulnerables a la extinción.

Aves: Se registraron un total de 38 especies de aves en la Reserva, pertenecientes a los ordenes Ciconiformes (1), Falconiformes (10), Charadriiformes (1), Columbiformes (2), Psittaciformes (2), Strigiformes (2), Apodiformes (1), Piciformes (2) y Passeriformes (17) (Tabla I). Del total de las aves registradas seis presentan problemas de conservación en el sur del país (SAG 2001): la torcaza (Patagioenas araucana Lesson) está en Peligro de extinción. La bandurria (Theristicus melanopis Gmelin), el halcón peregrino (Falco peregrinus Tunstall), el choroy (Enicognathus leptorhynchus King) y el carpintero negro (Campephilus magellanicus King) se catalogan como Vulnerables y el cóndor (Vultur gryphus Linné) se considera Rara. Nueve especies son endémicas a los bosques templados de Sudamérica, éstas son el choroy, la cachaña (E. ferrugineus Müller), el carpintero negro, el rayadito (Aphrastura spinicauda Gmelin), el comesebo grande (Pygarrhichas albogularis King), 
el Hued-hued (Pteroptochos tarnii King), el chucao (Scelorchilus rubecula Kittlitz), la viudita (Colorhamphus parvirostris Darwin) y el cometocino patagónico (Phrygilus patagonicus Lowe). Cabe destacar que hay un ave introducida en Chile que no había sido registrada en la provincia de Palena, la codorniz (Callipepla californica Shaw), que llegó hace aproximadamente 5-6 años, posiblemente de Argentina (información obtenida de los guardaparques). Esta especie no está incluida en la lista (Tabla I), ya que no se escuchó dentro del parque, pero sí se registró en las zonas campestres aledañas.

ANFIBIOS Y REPTILES: Sólo se detectaron anfibios en dos hábitats, correspondientes a los hábitats altos de la Reserva: el bosque de lenga-coigüe y el mallín aledaño, ambos en el sector del río Correntoso. En el bosque de lenga-coigüe se encontraron tres ejemplares adultos de rana grisácea (Batrachyla leptopus Bell) y un ejemplar adulto de rana jaspeada ( $B$. antartandica Barrio), mientras que en el sector del mallín del río Correntoso se detectaron larvas y adultos de sapo verrugoso (Alsodes verrucosus Phillipi) y sapito cuatro ojos (Pleurodema thaul Lesson), además de un ejemplar adulto de B. leptopus. El endemismo de los anfibios a los bosques templados de Sudamérica austral es total, la única excepción corresponde a Pleurodema thaul (sapito de cuatro ojos) que se distribuye hasta Chile central (Cei 1962). Para las categorías de conservación la mayoría de los anfibios están catalogados como "inadecuadamente conocidas" o Fuera de peligro. En cuanto a los reptiles, sólo se encontró un individuo de lagartija anaranjada (Liolaemus pictus Dumeril y Bibron) refugiado bajo piedras. Sin embargo, hay otra especie de lagarto descrita para la zona y que no fue observada en este muestreo, esta especie es la lagartija de vientre azul (Liolaemus cyanogaster Dumeril y Bibron). En el caso de los reptiles, L. pictus está en la categoría de vulnerable a la extinción para la zona sur.

\section{ANÁLISIS POR HÁBITAT}

De acuerdo al análisis por hábitat definido en la Reserva se observó que la mayor riqueza de especies ocurre en los bosques de lenga-coigüe con 39 especies (14 mamíferos, 23 aves y dos anfibios. Tabla I). Aquí aparecen especies como la huiña, la rata arbórea (Irenomys tarsalis Philippi), el roedor topo valdiviano (Geoxus valdivianus Philippi), el murciélago orejudo menor (Histiotus montanus Philippi \& Landbeck), la torcaza, el choroy, la cachaña, el carpintero negro, el comesebo grande, el chucao y el churrín (Scytalopus magellanicus Gmelin), la rana grisácea y rana jaspeada. Los registros de huemul en este sector se asociaron siempre a los cursos de agua (por ejemplo el río Correntoso). Este hábitat presenta el mayor endemismo de especies de aves, anfibios y micromamíferos. Se registraron seis especies de mamíferos con problemas de conservación; el huemul, el puma, el pudú, la huiña, el murciélago oreja de ratón, el zorro culpeo y tres aves; la torcaza, el choroy y el carpintero negro.

El hábitat de matorral mixto es el segundo hábitat de la Reserva con mayor riqueza total de especies, con 34 especies (Tabla I). En este hábitat se registran también especies de mamíferos con problemas de conservación como el huemul y el quirquincho de la Patagonia, incluso se encontraron varias galerías del quirquincho de la Patagonia (piche) en el área de matorral por debajo de la cota de la Reserva (700 msnm). En relación a las especies de aves destaca la alta riqueza de Falconiformes (8) y la disminución de especies endémicas al bosque templado a una sola, el cometocino patagónico. Se registran cinco especies con problemas de conservación, el zorro culpeo, el huemul, el pudú, la bandurria y el halcón peregrino (Tabla I). Pero también se registraron huellas de animales domésticos, vacuno, caballos y perros, pues este sector representa la zona de transición entre las praderas y campos que rodean la Reserva y el área protegida en sí.

En el hábitat denominado renoval de Nothofagus, la riqueza de especies totales se reduce a 28 y no hay evidencias de anfibios. El endemismo de aves es de cinco especies y en mamíferos de una. Las especies con problemas de conservación registradas aquí son el murciélago oreja de ratón, el zorro culpeo, el puma, la huiña, el huemul, la torcaza y el choroy (Tabla I).

En los sectores más altos de la Reserva con áreas de afloramientos rocosos que incluye además acantilados y bosques achaparrados de ñirres, la riqueza total de especies se reduce a 22 (Tabla I).

Destaca la alta riqueza de especies Falconiformes (9) y por ser el único hábitat con registro de vizcachas. Las huellas de felino encontradas en la zona de afloramientos rocosos (sobre los $1.000 \mathrm{msnm}$ ) 
Gayana 70(2), 2006

TABLA I. Lista de especies de vertebrados terrestres registradas en los diferentes hábitats de la Reserva Nacional Futaleufú.

TABLE I. Checklist of vertebrate species recorded in different habitats of National Reserve Futaleufú.

Especies registradas

\begin{tabular}{|c|c|c|c|c|c|c|c|c|}
\hline Clase & Orden & Nombre científico & Ciprés & $\begin{array}{l}\text { Matorral } \\
\text { mixto }\end{array}$ & $\begin{array}{c}\text { Bosque Coigue- } \\
\text { Lenga }\end{array}$ & $\begin{array}{l}\text { Renoval } \\
\text { Nothofagus }\end{array}$ & Mallín & $\begin{array}{c}\text { Rocas } \\
\text { expuestas }\end{array}$ \\
\hline \multirow[t]{18}{*}{ MAMALIA } & Quiroptera & Myotis chiloensis & $\mathrm{x}$ & $\mathrm{x}$ & $\mathrm{x}$ & $\mathrm{x}$ & $\mathrm{x}$ & \\
\hline & & Histiotus montanus & $\mathrm{x}$ & & $\mathrm{x}$ & & & \\
\hline & Rodentia & Oligoryzomis longicaudatus & & $\mathrm{x}$ & & $\mathrm{x}$ & $\mathrm{x}$ & $\mathrm{x}$ \\
\hline & & Abrothrix olivaceus & $\mathrm{x}$ & $\mathrm{x}$ & $\mathrm{x}$ & & & \\
\hline & & A. longipilis/sanbornii ${ }^{1}$ & & $\mathrm{x}$ & $\mathrm{x}$ & $\mathrm{x}$ & $\mathrm{x}$ & \\
\hline & & Geoxus valdivianus & & $\mathrm{x}$ & $\mathrm{x}$ & & $\mathrm{x}$ & \\
\hline & & Loxodontomys micropus & $\mathrm{x}$ & $\mathrm{x}$ & $\mathrm{x}$ & $\mathrm{x}$ & $\mathrm{x}$ & \\
\hline & & Irenomys tarsalis & $\mathrm{x}$ & & $\mathrm{x}$ & & & \\
\hline & & Lagidium viscacia & & & & & & $\mathrm{x}$ \\
\hline & Carnivora & Pseudalopex culpaeus & $\mathrm{x}$ & $\mathrm{x}$ & $\mathrm{x}$ & $\mathrm{x}$ & & $\mathrm{x}$ \\
\hline & & Conepatus humboltii & $\mathrm{x}$ & $\mathrm{x}$ & $\mathrm{x}$ & $\mathrm{x}$ & & \\
\hline & & Oncifelis sp.* & & & & & & $\mathrm{x}$ \\
\hline & & Oncifelis guigna & $\mathrm{x}$ & & $\mathrm{x}$ & $\mathrm{x}$ & & \\
\hline & & Puma concolor & $\mathrm{x}$ & & $\mathrm{x}$ & $\mathrm{x}$ & & \\
\hline & Edentata & Euphractus pichy & & $\mathrm{x}$ & & $\mathrm{x}$ & & $\mathrm{x}$ \\
\hline & Artiodactyla & Pudu puda & & $\mathrm{x}$ & $\mathrm{x}$ & & $\mathrm{x}$ & \\
\hline & & Hippocamelus bisulcus & & $\mathrm{x}$ & $\mathrm{x}$ & $\mathrm{x}$ & & $\mathrm{x}$ \\
\hline & Lagomorfos & Lepus capensis & & $\mathrm{x}$ & $\mathrm{x}$ & & & \\
\hline \multirow[t]{32}{*}{ AVES } & Ciconiformes & Theristicus melanopis & & $\mathrm{x}$ & & & & \\
\hline & Falconiformes & Vultur gryphus & & & & & & $\mathrm{x}$ \\
\hline & & Coragyps atratus & & & & & & $\mathrm{x}$ \\
\hline & & Geranoaetus melanoleucus & & $\mathrm{x}$ & & & & $\mathrm{x}$ \\
\hline & & Buteo polyosoma & & $\mathrm{x}$ & & & & $\mathrm{x}$ \\
\hline & & Circus cinereus & & $\mathrm{x}$ & & & & \\
\hline & & Milvago chimango & & $\mathrm{x}$ & & & & $\mathrm{x}$ \\
\hline & & Phalcoboenus albogularis & & $\mathrm{x}$ & & & & $\mathrm{x}$ \\
\hline & & Polyborus plancus & & $\mathrm{x}$ & & $\mathrm{x}$ & & $\mathrm{x}$ \\
\hline & & Falco peregrinus & & $\mathrm{x}$ & & & & $\mathrm{x}$ \\
\hline & & Falco sparverius & & $\mathrm{x}$ & & & & $\mathrm{x}$ \\
\hline & Charadriiformes & Vanellus chilensis & & $\mathrm{x}$ & & & & \\
\hline & Columbiformes & Patagioenas araucana & & & $\mathrm{x}$ & $\mathrm{x}$ & & \\
\hline & & Zenaida auriculata & & $\mathrm{x}$ & $\mathrm{x}$ & $\mathrm{x}$ & & \\
\hline & Psittaciformes & Enicognathus ferrugineus & & & $\mathrm{x}$ & $\mathrm{x}$ & & \\
\hline & & Enicognathus leptorhynchus & & & $\mathrm{x}$ & $\mathrm{x}$ & & \\
\hline & Strigiformes & Bubo virginianus & & & $\mathrm{x}$ & $\mathrm{x}$ & & \\
\hline & & Glaucidium Nahum & & & $\mathrm{x}$ & $\mathrm{x}$ & & \\
\hline & Apodiformes & Sephanoides sephaniodes & $\mathrm{x}$ & $\mathrm{x}$ & $\mathrm{x}$ & $\mathrm{x}$ & & $\mathrm{x}$ \\
\hline & Piciformes & Colaptes pitius & $\mathrm{x}$ & $\mathrm{x}$ & $\mathrm{x}$ & & & \\
\hline & & Campephilus magellanicus & & & $\mathrm{x}$ & & & \\
\hline & Passeriformes & Aphrastura spinicauda & $\mathrm{x}$ & & $\mathrm{x}$ & $\mathrm{x}$ & & \\
\hline & & Pygarrhichas albogularis & & & $\mathrm{x}$ & & & \\
\hline & & Pteroptochos tarnii & $\mathrm{x}$ & & $\mathrm{x}$ & & & \\
\hline & & Scelorchilus rubecula & $\mathrm{x}$ & & $\mathrm{x}$ & & & \\
\hline & & Scytalopus magellanicus & $\mathrm{x}$ & & $\mathrm{x}$ & & & \\
\hline & & Xolmis pyrope & $\mathrm{x}$ & $\mathrm{x}$ & $\mathrm{x}$ & $\mathrm{x}$ & & $\mathrm{x}$ \\
\hline & & Elaenia albiceps & $\mathrm{x}$ & $\mathrm{x}$ & $\mathrm{x}$ & $\mathrm{x}$ & $\mathrm{x}$ & $\mathrm{x}$ \\
\hline & & Colorhamphus parvirostris & & & $\mathrm{x}$ & $\mathrm{x}$ & & \\
\hline & & Tachycineta meyeni & & & $\mathrm{x}$ & & $\mathrm{x}$ & \\
\hline & & Pygochelidon cyanoleuca & & & & & $\mathrm{x}$ & \\
\hline & & Troglodytes aedon & $\mathrm{x}$ & $\mathrm{x}$ & $\mathrm{x}$ & $\mathrm{x}$ & $\mathrm{x}$ & $\mathrm{x}$ \\
\hline
\end{tabular}


Catastro de vertebrados terrestres, X Región. Elgueta, E. ET AL.

continuación Tabla I.

Especies registradas

\begin{tabular}{|c|c|c|c|c|c|c|c|c|}
\hline Clase & Orden & Nombrecientífico & Ciprés & $\begin{array}{l}\text { Matorral } \\
\text { mixto }\end{array}$ & $\begin{array}{l}\text { Bosque Coigue- } \\
\text { Lenga }\end{array}$ & $\begin{array}{l}\text { Renoval } \\
\text { Nothofagus }\end{array}$ & Mallín & $\begin{array}{c}\text { Rocas } \\
\text { expuestas }\end{array}$ \\
\hline & & Turdus falcklandii & $\mathrm{x}$ & $\mathrm{x}$ & $\mathrm{x}$ & $\mathrm{x}$ & $\mathrm{x}$ & \\
\hline & & Zonotrichia capensis & & $\mathrm{x}$ & & $\mathrm{x}$ & & $\mathrm{x}$ \\
\hline & & Curaeus curaeus & & & $\mathrm{x}$ & $\mathrm{x}$ & & \\
\hline & & Phrygilus patagonicus & $\mathrm{x}$ & $\mathrm{x}$ & $\mathrm{x}$ & $\mathrm{x}$ & $\mathrm{x}$ & $\mathrm{x}$ \\
\hline & & Diиса dіиса & & $\mathrm{x}$ & & & & \\
\hline & & Carduelis barbata & & $\mathrm{x}$ & $\mathrm{x}$ & $\mathrm{x}$ & $\mathrm{x}$ & $\mathrm{x}$ \\
\hline \multirow[t]{4}{*}{ AMPHIBIA } & Anura & Alsodes verrucosus & & & & & $\mathrm{x}$ & \\
\hline & & Batrachyla antartandica & & & $\mathrm{x}$ & & & \\
\hline & & Batrachyla leptopus & & & $\mathrm{x}$ & & $\mathrm{x}$ & \\
\hline & & Pleurodema thaul & & & & & $\mathrm{x}$ & \\
\hline REPTILIA & Squamata & Liolaemus pictus & & $\mathrm{x}$ & & & & \\
\hline \multicolumn{3}{|c|}{ Total de especies } & 20 & 34 & 39 & 28 & 17 & 22 \\
\hline
\end{tabular}

${ }^{[1]}$ Los molares encontrados no permiten la diferenciación entre A. longipilis y A. sanbornii, ambos descritos para la región.

*Potencial Oncifelis geofroyii. Ver discusión en texto

las hemos registrado inicialmente como Oncifelis sp., aunque no corresponde al hábitat característico de O. guigna, como se discutirá mas abajo. Sólo se registra una especie de ave endémica, el cometocino patagónico. Como especies con problemas de conservación destaca el registro sistemático de vizcachas y huemul desde la instauración del área protegida (informe interno CONAF) además de los Falconiformes cóndor y halcón peregrino.

En los bosquetes de ciprés, los cuales sólo presentan un estrato vegetal y son monoespecíficos, el número de especies de vertebrados fue 20 (Tabla I ). Respecto a los mamíferos los bosques de coníferas muestran el segundo registro de Histiotus montanus, en el ecotono con el matorral mixto o praderas fuera de la Reserva. Las especies de aves endémicas al bosque templado presentes en este hábitat son cuatro, el rayadito, el hued-hued del sur, el chucao y el cometocino patagónico y los mamíferos son las dos especies de murciélagos y la rata arbórea (Tabla I). No hay evidencias de especies con problemas de conservación para las aves pero sí los registros de pumas, zorros culpeos y murciélagos agregan tres especies a este hábitat.

Por último, el hábitat denominado mallín presentó la menor riqueza de especies totales, 17 especies (siete mamíferos, siete aves y tres anfibios, Tabla I), sin embargo, fue el único hábitat donde se registró a los anfibios $P$. thaul y A. verrugosus (Tabla I).

\section{DISCUSION}

\section{ESPECIES POTENCIALES}

El primer objetivo de este estudio fue entregar un catastro de los vertebrados terrestres presentes en la Reserva de Futaleufú, dando cuenta de un mínimo de especies detectadas. En el caso de las especies raras o de baja incidencia en los muestreos (mustélidos y felinos), muestreos más prolongados o abarcando diferentes estaciones climáticas posiblemente darían cuenta de otras especies no registradas en este estudio o confirmarían las registradas por evidencias indirectas. Por ejemplo, respecto a las huellas de felino encontradas en galerías de la zona de afloramientos rocosos y que anotamos como Oncifelis sp., proponemos esta especie como el gato de Geoffroy o gato montés argentino (Oncifelis geofroyi Orbigny \& Gervais) debido a que sus huellas fueron encontradas en un hábitat alejado de los bosques y porque los propios guardaparques han diferenciado por coloración y hábitats claramente a dos especies de felinos en la Reserva, incluso describen a la huiña como "la otra especie que anda en el monte" y a diferencia del felino que "ronda las partes altas". Estos antecedentes se apoyan además con las capturas del gato de Geoffroy realizadas por Lucherini (2001) en el Parque Argentino "Los Alerces", tan sólo a 50 km del paso Futaleufú. 
El numero de micromamíferos podría aumentar si mediante trampeos extensivos se pudiera confirmar la presencia de tres especies de roedores con escasos registros en Chile y que no fueron incluidos en estas listas. Eligmodontia morgani (Allen) y E. typus (Cuvier) sólo tienen tres registros en Chile en la Cordillera de los Andes sobre las latitudes $38^{\circ} 30^{\prime} \mathrm{S}$, $45^{\circ} 20^{\prime} \mathrm{S}$ y $51^{\circ} 10^{\prime} \mathrm{S}$ (Lago Icalma, Coyhaique y PN Torres del Paine respectivamente). En la estepa argentina el género Eligmodontia llega a ser abundante (Pearson 1993) pero no existen muestreos anteriores en el área de Futaleufú. Otra especie cuya distribución no está confirmada aún es Phyllotis xanthopygus (Waterhouse) presente en los Andes argentinos y con registro en Chile en las áreas adyacentes al lago General Carrera (IX Región) (Muñoz-Pedreros \& Yáñez 2000).

En relación a las aves, dos especies raras residentes en la zona y asociadas a bosques de Nothofagus, pero que no fueron evidenciadas directamente en la reserva, son el peuquito (Accipiter bicolor Vieillot) y el aguilucho de cola rojiza (Buteo ventralis Gould). Otras especies como la lechuza (Tyto alba Scopoli) y el concón (Strix rufipes King) también han sido descritas para esta región, pero sus grandes ámbitos de hogar y hábitos nocturnos podrían explicar su ausencia en nuestros muestreos (Jaramillo et al. 2003).

En el caso de los anfibios la baja representación puede deberse a la geomorfología de la Reserva, la que presenta muy pocos hábitats potenciales que mantengan las condiciones de humedad y sitios de reproducción que éstos requieren, aunque pudiera incluirse a la especie Eupsophus calcaratus Günther, que fue observada fuera de la Reserva (río Chico) en la cota $500 \mathrm{msnm}$. Por otra parte, otra especie descrita para la zona corresponde a Bufo rubropunctatus Guichenot. Aunque la presencia de este anfibio no ha sido registrada en las localidades descritas para Chile, en estos últimos años (M. Méndez obs. pers.), ésta ha sido encontrada en el antes mencionado Parque Nacional Los Alerces en Argentina (Vidoz \& Ubeda 2000). De la misma manera, el caso de detectar sólo una especie de reptil se explicaría por las condiciones ambientales en el momento del muestreo (baja temperatura y nubosidad) cuando la actividad de estos reptiles se ve reducida, y por lo tanto el muestreo para estas especies estaría sub-representado en el área de estudio.

\section{ESTRUCTURA DEL HÁBITAT Y RIQUEZA}

En relación al análisis de riqueza de especies y endemismos por hábitat en el bosque de lenga-coigüe se registró la mayor riqueza para las aves junto con el mayor endemismos para mamíferos, lo que podría deberse a la mayor heterogeneidad del ambiente dentro de este hábitat, dada por un dosel multiestratificado, la presencia de grandes árboles, troncos muertos y parches de sotobosque denso (Díaz et al. 2005). Por ejemplo, el chucao y el churrín necesitan sotobosque para alimentarse, refugiarse y nidificar (Sieving et al. 2000; Reid et al. 2004; Díaz et al. 2005), el carpintero negro, el rayadito y el comesebo grande requieren de bosque primario y/o presencia de árboles grandes muertos en pie (Fjeldså \& Krabbe 1990; Díaz et al. 2005). A su vez $O$. guigna es una especie con una marcada preferencia por el este tipo de hábitat (Nowell \& Jackson 1996; Acosta-Jamett \& Simonetti 2004). Al mismo tiempo, estas especies son sensibles a la fragmentación de hábitat, lo que significa que no habitan en fragmentos de bosque de superficies muy pequeñas (Willson et al. 1994). De las especies endémicas al bosque templado, la cachaña, el carpintero negro, el comesebo grande, la viudita y la huiña han sido catalogadas como las especies que presentan mayor amenaza, dado sus reducidas abundancias, rangos geográficos pequeños y alta especificidad de hábitat (Cofré 1999; Cofré \& Marquet 1999).

El hábitat de matorral mixto es el segundo hábitat con mayor riqueza de especies, ya que aquí se conjugan especies de aves y mamíferos que habitan tanto matorral nativo como hábitats más abiertos (por ejemplo chincol (Zonotrichia capensis Müller) y la diuca (Diuca diuca Molina)), dando por resultado una zona de transición entre el matorral y praderas fuera de la Reserva y el área de renovales (ver polígonos en Figura 1).

La menor riqueza de especies de aves y roedores endémicos en el hábitat denominado renoval de Nothofagus se puede deber a una menor heterogeneidad estructural, ya que los ejemplares de Nothofagus presentaban la misma clase etaria (crecimiento posterior a un incendio), no había presencia de grandes árboles, ni troncos y no había sotobosque. La ausencia de tales estructuras podría explicar la ausencia del pitío (Colaptes pitius Molina), el carpintero negro, el comesebo grande, el hued-hued del sur, el chucao y el churrín que usan 
estas estructuras para descansar, alimentarse y nidificar (Díaz et al. 2005).

En relación al hábitat de afloramientos rocosos con bosques achaparrados de ñirres, aunque el número de especies totales fue menor que en los hábitats de bosque, reviste alta importancia por presentar lugares para la reproducción de especies con problemas de conservación o raras y que son encontradas allí, lejos de las áreas de influencia de los campos agrícolas que rodean la Reserva (Ej. vizcachas, quirquinchos (o piche) y huemules). De la misma manera los acantilados proveen sitios de nidificación para las aves rapaces, como cóndores, el halcón peregrino y el carancho cordillerano del sur (Phalcoboenus albogularis Gould).

Por último, el mallín a pesar de su baja representatividad en superficie y riqueza de especies corresponde a un hábitat importante para la conservación de anfibios en esta región, debido a que es en este lugar donde ocurren los eventos de reproducción de la mayoría de las especies registradas.

\section{AMENAZAS PASADAS, PRESENTES Y FUTURAS}

Es importante que cualquier área protegida mantenga la concepción teórica por la cual fue diseñada, donde las especies silvestres estarían libres de las amenazas que afectan su sobrevivencia (cacería, interacción con especies exóticas y destrucción del hábitat). A pesar que en este estudio se logró confirmar la presencia de mamíferos y aves con problemas de conservación, alto endemismo y/o raras que aún sobreviven en el área, esto no asegura su sobreviviencia poblacional a largo plazo dentro de la Reserva, ya sea por procesos estocásticos asociados a los bajos tamaños poblacionales (Mella et al. 2002) o a los diversos riesgos potenciales en esta región, entre los que podemos mencionar: (1) sensibilidad poblacional a la fragmentación del hábitat, por ejemplo la riqueza de especies de aves disminuye en fragmentos pequeños y la composición de especies cambia (Willson et al. 1994; Estades \& Temple 1999; Cornelius et al. 2000), y de las especies que subsisten, la superficie existente de hábitat puede ser insuficiente para mantener una población viable a largo plazo. En el caso de los mamíferos chilenos un análisis teórico del área mínima para retener poblaciones viables dentro de áreas protegidas de Chile (Simonetti \& Mella 1997) muestra que para el huemul se requieren entre 28.000-2.500.000 hectáreas de hábitat, según el cálculo con rangos de densidad conocidos (Povilitis 1986). Valor que supera las 12.064 hectáreas de la Reserva; (2) La introducción de animales domésticos dentro de la Reserva, ya que no existe un límite claro con los campos aledaños, se traduce en la posible trasmisión de enfermedades desde el ganado a los huemules, siendo la segunda causa de extinción de los huemules (Povilitis 1998), a su vez los perros asilvestrados ejercen una presión de depredación sobre los animales jóvenes (Povilitis 2002). En Argentina, en el Parque Los Glaciares Prov. de Sta. Cruz, investigadores de la Fundación Vida Silvestre Argentina (2002) mencionan casos de depredación ejercida por perros asilvestrados sobre ejemplares de huemul. (3) Las especies exóticas como jabalíes, visones y liebres también presentes en el área protegida modifican el hábitat para las especies silvestres, o las depredan directamente. (4) La captura de mamíferos nativos para el mercado ilegal de pieles y armazones aún es una amenaza que no ha podido ser fiscalizada de manera cabal. Cabe recordar que a pesar que Chile ha suscrito nueve convenios internacionales respecto a la regulación de la comercialización de la fauna silvestre (siendo el más relevante el de CITES) y la protección total desde 1993 por la promulgación del reglamento de ley de caza $\mathrm{N}^{\circ}$ 4.601, la explotación de algunos mamíferos ha continuado de manera ilegal como lo muestran los decomisos permanentes de pieles de zorros (Pseudalopex spp.), felinos (Oncifelis geofroyi), nutrias (Lontra spp.) y chingues (Conepatus spp.) hacia Esquel por el paso fronterizo de Futaleufú y Río Gallegos (Iriarte 2000).

A pesar de todo, a nivel regional la protección de esta Reserva ayudará a mantener la conectividad a nivel de las metapoblaciones, pero en futuras estrategias de planificación local se debe propiciar la mantención de la heterogeneidad del paisaje y el control de las especies exóticas en esta área protegida para asegurar la factibilidad y permanencia en el tiempo de la actual diversidad. Por ejemplo con la protección de elementos y estructuras claves para algunas especies dentro de los bosques de la Reserva como la mantención de árboles grandes, el sotobosque y los troncos caídos, ayudaría a mantener las poblaciones de las aves que dependen de este tipo de hábitat, asociado a monitoreos de la dinámica de las poblaciones de especies recientemente introducidas en esta zona, 
como jabalíes, visones y codornices (de la cual no existen estudios al respecto en la zona), porque no se sabe cómo estas especies podrían estar afectando a especies nativas con requerimientos de hábitat similares.

\section{AGRADECIMIENTOS}

Agradecemos a CONAF X Región la posibilidad de publicar los datos obtenidos durante el estudio de línea base de la fauna realizado por la Fundación Senda Darwin para la elaboración del Plan de Manejo de la Reserva Nacional Futaleufú. Este trabajo fue financiado por la Fundación Raíces.

Se agradece también la ayuda prestada por los guardaparques Sres. Evaristo Araneda y Néstor Diocarets y a nuestros amigos y asistentes de terreno los biólogos Luis Espinoza y José J. Núñez. Se agradece por su apoyo constante al Sr. José Alvarez de la oficina de la UGPS-CONAF Puerto Montt.

“C. Smith-Ramírez agradece a la Fundación BBVA (Spain), Fondecyt-Fondap 1501-0001 (Programa 3) Centro de estudios avanzados en ecología y biodiversidad de la P. Universidad Católica de Chile y al Proyecto Nzcleo Milenio N: 99 - 103 FICM".

\section{BIBLIOGRAFIA}

Acosta-Jamett G. \& J.A. Simonetti. 2004. Habitat use by Oncifelis guigna and Pseudalopex culpaeus in a fragmented forest landscape in central Chile. Biodiversity and Conservation 13: 1135-1151.

Cofre, H. L. 1999. Patrones de rareza de las aves del bosque templado de Chile: implicancias para su conservación. Boletín Chileno de Ornitología 6: 8-16.

Cofré, H. \& P. MARQuet. 1999. Conservation status, rarity, and geographic priorities for conservation of Chilean mammals: an assessment. Biological Conservation 88: 53-68.

Convention on International Trades in ENDANGERED SPECIES OF WILD FAUNA AND FLORA (CITES). 2004. Appendices I-IIII, Official Documents.http:// www.cites.org/eng/disc/text.shtml

Cornelius, C., H. Cofré \& P.A. Marquet. 2000. Effects of habitat fragmentation on bird species in a relict temperate forest in semiarid Chile. Conservation Biology 14: 534-543.

Díaz, I., J.J. Armesto, S. Reid, K.E. Sieving \& M.F. WILLSON. 2005. Linking forest structure and composition: avian diversity in successional forests of Chiloé Island, Chile. Biological Conservation 123: 91-101.

Di CASTRi \& E. HAYeK. 1976. Bioclimatología de Chile. Imprenta Editorial Universidad Católica, Santiago.

Estades, C.F. \& S.A. Temple. 1999. Deciduous-forest bird communities in a fragmented landscape dominated by exotic pine plantations. Ecological Applications 9: 573- 585.

Fueldsa, J. \& N. Krabbe. 1990. Birds of the high Andes. Zoological Museum, University of Copenhagen, Copenhagen.

Freemark, K.E. \& H.G. Merriam. 1986. Importance of area and habitat heterogeneity to bird assemblages in temperate forest fragments. Biological Conservation 36: 115-142.

Fundación Vida Silvestre Argentina. 2002. Bosques y selvas. Editado 2002 http//www.vidasilveste.org.ar/bosques/.

Hutto, R.S., S.M. Pletschet \& P. Hendricks. 1986. A fixed-radius point count method for non-breeding and breeding season use. Auk 103: 593-602.

Iriarte, A. 2000. Conservación de mamíferos en Chile. En: Mamíferos de Chile (eds.). Muñoz-Pedreros A. \& J. Yáñez CEA Ediciones. Valdivia.

Iriarte A., J. Jiménez, L. Contreras \& F. Jaksic. 1989. Small-mammal availability and consumption by the fox, Dusycion culpaues, in Central Chilean scrublands. Journal of Mammalogy 70(3): 641-645.

Jaramillo, A., P. Burke \& D. Beadle. 2003. Birds of Chile including the Antarctic Peninsula, the Falkland Islands and South Georgia. Christopher Helm, London, $240 \mathrm{pp}$.

KerR J.T., \& L. PACKER. 1997. Habitat heterogeneity as a determinant of mammal species richness in high energy regions. Nature 385: 252-254.

LUCHERINI, M. 2001. The kod-kod (Oncifelis guigna) and the Patagonia mountain forest: linking two conservation priorities. First report GECM. (Accessed January 2002) http:// carnivorecology.free.fr/archives2002.htm

Martínez D., J. Rau, R. Murúa \& M. Tillerías. 1993. Depredación selectiva de zorros chillas (Psudalopex griseus) en la pluviselva valdiviana, Chile. Revista Chilena Historia Natural 66:419-426.

Mella J., J. Simonetti, A. Spotorno \& L. Contreras. 2002. Mamíferos de Chile. En: Diversidad y conservación de los mamíferos Neotropicales. Ceballos G. \& J. Simonetti Editores. CONABIO-UNAM, México, $582 \mathrm{pp}$.

MuÑoz-Pedreros, A. \& J. YÁÑEz (eds). 2000. Mamíferos de Chile. CEA Ediciones-Valdivia.

Myers N., R.A. Mittermeir, C.G. Mittermeir, G.A. FonseCA \& J. Kent. 2000. Biodiversity hotspots for conservation priorities. Nature 403: 853-858.

Nowell K. \& P. JACKSON. 1996. Wild cats: Status Survey and Conservation Plan. IUCN, Gland. Switzerland.

Primack, R., R. Rozzi, P. Feinsinger, R. Dirzo \& F. MASSARDO. 2001. Fundamentos de conservación 
biológica: Perspectivas latinoamericanas. Fondo de Cultura Económica, Ciudad de México. 797 pp.

Pearson, O. 1983. Characteristics of a mammalian fauna from forests in Patagonia, Southern Argentina. Journal of Mammalogy 64(3): 476-492.

Pearson, O. 1995. Annotated keys for identifying small mammals living in or near Nahuel Huapi National Park or Lanin National Park, southern Argentina. Mastozoología Neotropical 22: 99-148.

PoviLitis, A. 1986. Huemuls in areas adjacent to glaciers in southern Chile. Mountain Chronicles: 273-275.

Povilitis, A. 1998. Characteristics and conservation of a fragmented population of huemul (Hippocamelus bisculus) in central Chile. Biological Conservation 86: $97-104$.

Povilitis, A. 2002. El estado actual del huemul (Hippocamelus bisulcus) en Chile central. Gayana. 66 (1): 59-68.

Prendergast, B. 1993. Rare species, the coincidence of diversity hotspots and conservation strategies. Nature 365: 335-337.

Ralph, C., G. Geupel, P. Pyle, T. Martin \& D. Desante. 1993. Handbook of field methods for monitoring landbirds. Pacific Southwest Research Station Albany, California.
Reid, S., I.A. Díaz, J.J. Armesto \& M.F. Willson. 2004. Importance of native bamboo for understory birds in Chilean temperate forests. Auk 121(2): 515525.

SAG. 2001. Cartilla de Caza. Documento técnico. Departamento de Protección de los Recursos Naturales Renovables. Cuarta Edición, Santiago. Depto. Comunicaciones, Servicio Agrícola y Ganadero.

Sieving, K.E., M.F. Willson, \& T. L. De Santo. 2000. Defining corridor functions for endemic birds in fragmented south-temperate rainforest. Conservation Biology 14: 1120-1132.

Simonetti, J.A. 1999. Diversity and conservation of terrestrial vertebrates in Mediterranean Chile. Revista Chilena de Historia Natural 72: 493-500.

Simonetti, J. \& J. Mella. 1997. Park size and the conservation of Chilean mammals. Revista Chilena Historia Natural 70: 213-220.

Vidoz, F. \& C.A. ÚBEDA. 2000. Bufo rubropunctatus Guichenot en Argentina: Registros recientes y nuevos hábitats para la especie. Boletín Sociedad de Biología. Concepción 71: 49-52.

Willson, M.F., T.L. De SAnto, C. SABAG, \& J.J. Armesto. 1994. Avian communities of fragmented southtemperate rainforest in Chile. Conservation Biology 8: 508-520.

Fecha de recepción: 17.08.05

Fecha de aceptación: 04.10.05 